\title{
El trabajo social uruguayo y sus bases críticas
}

\section{The Uruguayan Social Work and its critical bases}

\author{
José Fernando Siqueira da Silva ${ }^{a}$ \\ (- http://orcid.org/0000-0003-1040-9558 \\ José Pablo Bentura ${ }^{b}$ \\ (D) http://orcid.org/0000-0003-2341-4751
}

\begin{abstract}
Resumen: Este artículo tiene como objetivo debatir la génesis, el desarrollo y la consolidación de la fracción más crítica del Trabajo Social en Uruguay. Producto de un estudio postdoctoral realizado en la Universidad de la República y financiado por la FAPESP en 2020, el texto aborda los elementos centrales que apoyaron la formación de un Trabajo Social progresista a partir de las luchas colectivas latinoamericanas de las décadas de 1960, 1970 y 1980 y fin de la dictadura cívico-militar en Uruguay en 1985.
\end{abstract}

Palabras clave: Trabajo Social Uruguayo. Tendencias críticas. América Latina.

\begin{abstract}
This article aims to discuss the genesis, development and consolidation of the most critical fraction of Social Work in Uruguay. Product of a postdoctoral study conducted at the University of the Republic and funded by FAPESP in 2020, the text addresses the central elements that sustained the formation of a progressive Social Work from the Latin American struggles collectively fought in the 1960s, 1970 s and 1980s and the end of the civicmilitary dictatorship in Uruguay from 1985.
\end{abstract}

Keywords: Uruguayan Social Work. Critical trends. Latin America. 


\section{Observaciones iniciales}

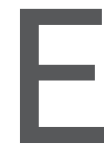

1 texto que aquí se presenta es producto de un estudio realizado

en 2020 durante una pasantía postdoctoral de 11 meses en la Universidad de la República (Udelar-Uruguay). Circunscritas al límite de páginas establecido para la presentación de este manuscrito, las siguientes páginas analizan brevemente aquellos elementos que determinaron la génesis, desarrollo y consolidación de la fracción más crítica del Trabajo Social en ese país. La investigación, sistematizada por el profesor investigador, contó con el apoyo de la FAPESP (proceso 2019/ 10340-0) y el aporte de su tutor, quien comparte la autoría de este texto.

\section{El Uruguay y sus particularidades en América Latina}

La trayectoria de Uruguay reafirmó la tradición dependiente (Fernandes, 2009; Marini, 2008; Katz, 2020), cuya base económica es predominantemente agroexportadora y constituida por grupos económicos cuyos intereses se enfocan fuera del país. Su formación social estuvo marcada por la fuerte participación de trabajadores inmigrantes europeos (Acosta, 2005, p. 27-28), aunque ha convivido con formas de producción arcaicas de perfil oligárquico, con trabajo esclavo (seguramente más tímido en comparación con otras regiones de América Latina) y con la penetración del capital monopolista (particularmente en las primeras décadas del siglo XX). Así, reafirmó, de distintas formas, su tradición exportadora, sin el desarrollo de industrias productoras de bienes, pero con inversiones internacionales que modernizaron paulatinamente sectores estratégicos para las exportaciones del país, como la industria frigorífica.

La economía uruguaya no constituyó el modelo económico que en América Latina es conocido como "enclave", es decir, no se sostuvo sustancialmente - como en los casos brasileño, mexicano y argentino (sin perjuicio de las diferencias en estos procesos) - en la masiva atracción de recursos externos para la inversión en la industria local, entre otras 
cosas porque el mercado interno uruguayo siempre ha sido poco atractivo. Por tanto, se consolidó una economía "orientada al exterior", pero con una base económica cuya propiedad estaba "controlada nacionalmente" (Real de Azua, 1984, p. 93-94). Sobre estas bases se conformó el tradicional sistema bipartidista, dividido entre el Partido Colorado ("colorados") y el Partido Nacional ("blancos"), que persistió hasta 1971 con la fundación del Frente Amplio, con una fuerte capacidad de absorber demandas de diversos sectores sociales, acomodar los intereses de las clases y sus diversos segmentos, además de articularse con una tradición caudillista y populista heterogénea (Real de Azua, 1984, p. 92).

Uruguay tiene una fuerte tradición republicano-liberal, luego reforzada por una perspectiva republicano-democrática más radical y bastante peculiar, especialmente a partir del primer batllismo (19031931). Esto impactó sustancialmente a la sociedad uruguaya desde temprana edad, proceso en el que también influyó la búsqueda de una identidad nacional marcada por el vacío interior y la presencia constante de fronteras con dos gigantes latinoamericanos: Brasil y Argentina. Los estudios de Gerardo Caetano (2015) revelan aspectos de una sociedad cuyas tensiones estructurales fueron amortiguadas (en términos de Real de Azua, 1984):

a) cierto tipo de ciudadanía "hiperintegradora", aunque también ha reproducido la tradicional división espacio rural/ciudad;

b) el intento de disputar el discurso y la práctica liberales, en el campo republicano explícito;

c) el énfasis en las reformas cívicas (nación/ciudadanía) y políticas (expresadas en formalismo constitucional), proceso este consolidado en la relación entre parlamento y ciudadanía;

d) la "reforma moral" y su consecuencia explícitamente laica y avanzada, que también tuvo una tradición española menos incidente (más tardía) en la banda oriental, en comparación con otras regiones colonizadas; 
e) una educación pública igualmente laica y absolutamente prematura para los estándares latinoamericanos, que implicó la creación de escuelas primarias en la segunda mitad del siglo XIX a través de la Ley de Educación Común (1877), propuesta por el entonces director de Instrucción Pública José Pedro Varela, durante la dictadura del coronel Lorenzo Latorre;

f) la influencia explícita de los valores e ideas francesas de finales del siglo XIX, con una base republicana, ilustrada, en apoyo de la ciencia y las artes, con una clara separación entre Estado e Iglesia. Esta orientación se expresó, por ejemplo, en las propuestas de importantes cuadros intelectuales ubicados entre finales del siglo XIX y las primeras décadas del XX, como Carlos Vaz Ferreira (republicano liberal) y José Enrique Rodó (positivista convencido), ambos de fuerte ascendencia grecolatina y representantes del "pensamiento vivo de la filosofía uruguaya” (Acosta, 2005, p. 42-47). Un pensamiento esencialmente plural, a veces ecléctico, así como una práctica pragmático-científica, de base liberal;

g) la relativa marginalidad de los sectores militares absorbidos originalmente por la fuerza del partido, la orientación republicana y la movilización de los sectores sociales.

La importancia del primer batllismo en la sociedad uruguaya es indiscutible. Esta tendencia, liderada por el Partido Colorado, marcó profundamente a Uruguay en los primeros 30 años del siglo XX. Precedió a la crisis del capital de 1929, la revolución bolchevique de 1917, comenzó antes de la Primera Guerra Mundial y se formó antes del Estado de Bienestar keynesiano. ${ }^{1}$ El reformismo batllista insistió en construir un país sólido, pequeño, industrial, moderno, un modelo de protección

\footnotetext{
Además de los dos gobiernos de José Batlle y Ordóñez (1903-1907 y 1911-1915), el pleno dominio del Partido Colorado fue sostenido por otros gobiernos: Claudio Williman (1907-1911), Feliciano Viera (1915-1919), Baltasar Brum (1919-1923), José Serrato (1923-1927) y Juan Campisteguy (1927-1931).
} 
social para los más débiles, atractivo para la mano de obra extranjera, y una alternativa económica y social a Buenos Aires.

Las reformas sociales, siempre bajo la presión de la clase trabajadora, se concentraron en algunas áreas estratégicas: ${ }^{2}$ a) asistencia pública; b) educación laica gratuita y obligatoria de primer nivel; c) jornada laboral de 8 horas diarias (1915); d) ley de descanso semanal (1920); e) sistema de pensiones, como los fondos de trabajadores/empleados del servicio público (1919) y la organización de pensiones de los trabajadores bancarios (1925). También es significativo destacar la ley del divorcio basada en la sola voluntad de la mujer (1913), así como la densidad y expansión de los cursos que ofrece la Universidad de la República con participación directa de los estudiantes. ${ }^{3}$ Cabe señalar que es en esta época (1927) que el Servicio Social tiene su génesis en el país articulado a las actividades realizadas por las “Visitadas Sociales de Higiene” (Ortega, 2008, p. 48).

La crisis mundial de 1929 impactó a Uruguay, pero no fue suficiente para derrotar al Partido Colorado, que históricamente había ocupado el poder ejecutivo nacional. El gobierno de Gabriel Terra, elegido en 1930 que asumió en 1931, incapaz de lidiar con las tensiones impuestas y los perversos efectos económicos de 1929, se convirtió en dictadura en 1933. Como destaca Real de Azúa (1984, p. 65, p. 65), se evocaron argumentos históricos ampliamente utilizados por los sectores más conservadores: exceso de gasto público, fuerte carga fiscal, déficit de la balanza comercial, la ruina de la producción agraria agudizada por la crisis de 1929, los tradicionales llamamientos anticomunistas, el exceso de huelgas caracterizadas como anarquistas, el peligro que tradicionalmente se atribuye a estos hechos. Estos argumentos se tradujeron en una amplia

2 Como referencias para este debate, véanse Caetano (2015) o Acosta (2005). La organización obrera uruguaya y sus primeras huelgas fueron influenciadas directamente por inmigrantes franceses y españoles, movimientos inspirados en la Comuna de París de 1871 y la Revolución Cantonal de 1873, de inspiración anarquista.

3 La fundación de la Universidad de la República tuvo lugar entre 1833 y 1849. La Federación de Estudiantes Universitarios del Uruguay (FEUU) tuvo sus orígenes en 1929. 
represión a estudiantes, políticos de izquierda, dirigentes sindicales, entre otros sectores de oposición.

El ímpetu reformista del Primer Batllismo se mantendrá en el neobatllismo, sumado a una política industrial más intensa. Esta intensificación se producirá paulatinamente con el fin de la dictadura en 1938, pero se intensificará sobre todo entre 1948 y 1958, período en el que el Segundo Batllismo intensifica su influencia dentro del Partido Colorado. ${ }^{4} \mathrm{Si}$ bien hubo aspectos que unieron el "populismo" uruguayo, argentino y brasileño (aunque a "posteriori”, Real de Azua, 1984, p. 60), es importante resaltar, al mismo tiempo, importantes diferencias. Entre los puntos en común se encuentran las claras políticas de industrialización y redistribución de los logros de la guerra y la política de sustitución de importaciones con fuerte participación estatal, así como la movilización de los sectores sociales vinculados a este proceso y beneficiados por él.

Por otro lado, si bien la política de conciliación de clases ha sido la base sustentadora del proyecto de desarrollo industrial y social, el giro distributivo uruguayo hacia los más pobres no tuvo el mismo impacto en los sectores dominantes que en sus vecinos, ni fue marcado por un fuerte impulso nacionalista (como sucedió, sobre todo, en Argentina o incluso en Brasil en los años cincuenta y principios de los sesenta). Además, como insiste Real de Azúa (1984), Luis Batlle era más un líder de partido que un líder al estilo de Perón o Vargas (o, en menor medida, Kubitschek-Goulart). Aquí, la tradición liberal-republicano-radical, apoyada por los partidos, determinó que la versión "populista" de este país no expresara las tendencias "justicialistas" de Argentina ni las tendencias "laborales" de Brasil. Es importante señalar aquí que, durante este período (1954), no es casualidad que el centro de formación en Trabajo Social

4 Juan José de Amégaza (marzo de 1943-1947), Luis Batlle Berres (marzo de 1947-1952, conocido como el fundador del neobatllismo) y el Consejo de Gobierno Nacional de Colorado (marzo de 1952-1959 - presidido por el mismo Luis Batlle Berres). Data de este período (1955), inicio de la renovación del PCU bajo el liderazgo de Rodney Arismendi (1913-1989), cuadro importante del Partido Comunista en el país, incluso para la fundación del Frente Amplio (FA) en 1971. 
estuviera vinculado al Ministerio de Instrucción Pública, absorbido por la Escuela Universitaria de Servicio Social (EUSS, 1958-1992).

El fin del neobatllismo y la primera victoria del Partido Nacional en 1958 culminó con la formación del Consejo Nacional de Gobierno liderado por los nacionalistas - por primera vez en el país - en marzo de 1959. Aquí, notamos algo importante que se impone como un rasgo común en toda América Latina: la caída de los valores internacionales de los productos primarios a fines de la década de 1950 (especialmente la carne y la lana, en el caso uruguayo) asociada al crecimiento de los productos importados (que generó un desequilibrio en la balanza comercial), el mantenimiento de la estructura de propiedad de la tierra heredada del período colonial, la desindustrialización, el acelerado proceso inflacionario y la inestabilidad del sector financiero en el país, aspectos que debilitaron al neobatllismo, desarmaron e hicieron inviable su proyecto de gobierno. La fórmula presentada como remedio es conocida: desestatización, privatización y apoyo al sector agrícola (Acosta, 2005, p. 72-74). Esta propuesta se expresó políticamente en lo que se ha dado en llamar "herrerismo", es decir, la alianza entre el ruralismo del carismático caudillo Benito Nardone y el líder Luis Alberto de Herrera del Partido Nacional.

El período previo al golpe cívico-militar estuvo marcado por una intensa movilización que involucró a estudiantes, intelectuales, sindicatos, fracciones de las clases medias (donde se insertaba la guerrilla tupamara) y trabajadores, una articulación expresiva que incidió en distintas generaciones y niveles sociales. La derecha, a su vez, estuvo compuesta por sectores rurales, el sector industrial, sectores urbanos medianos y de servicios, así como por la apatía de parte de los sectores populares. La forma en que se produjo el golpe fue igualmente original: exigió una articulación constante con los jefes civiles del Ejecutivo apoyados por las Fuerzas Armadas, con la participación del presidente electo colorado-ruralista Juan María Bordaberry Arocena (marzo de 1972/septiembre de 1973); continuó con su sucesor Colorado Pedro Alberto Demicheli Lizaso 
(quien permaneció poco más de dos meses en este cargo); así como consolidado con Aparicio Méndez, presidente de facto durante el régimen militar (este miembro de la extrema derecha del Partido Nacional). La función de este gobierno fue explícita: reafirmar el modelo dependiente expresado en el imperialismo estadounidense en la región con el fin de cualquier proyecto mínimamente independentista, eliminar a la oposición, promover un control social de las desigualdades y prevenir la propagación de culturas rebeldes y acciones de fomento del "liderazgo subversivo" (principalmente de orientación marxista-comunista).

La novedad político-partidaria en Uruguay se anunció en 1971 con la creación del Frente Amplio (FA), que aglutinó a sectores progresistas de diversas denominaciones y se mantuvo en la oposición durante el régimen militar. El proceso de redemocratización política a partir de marzo de 1985 reafirmará la hegemonía de los partidos Colorado y Nacional con tres gobiernos colorados (dos de ellos liderados por Julio María Sanguinetti - 1985/1990 y 1995/2000 - y uno por Jorge Batlle — 2000/2005) y uno gobierno blanco (dirigido por Luis Alberto Lacalle - 1990/1995). ${ }^{5}$ Tales asociaciones ya compartían el espacio político con el Frente Amplio, que creció significativamente en esos 20 años y estableció una importante hegemonía progresista entre marzo de 2005 y marzo de 2021 con Tabaré Vázquez (dos administraciones) y José Mujica. El proceso se vio nuevamente interrumpido con la elección de Lacalle Pou y su toma de posesión a partir de marzo de 2020, que llevo nuevamente al Ejecutivo al Partido Nacional desde un amplio frente conservador que articuló varias orientaciones y promovió una polémica coalición nunca antes vista en el Uruguay: blancos y colorados en el mismo bando (además del "Partido Independiente", el "Partido de la Gente" y la extrema derecha presente representada por el partido “Cabildo Abierto").

5 Es importante señalar que fue durante este período (1992), en el grupo de luchas por la redemocratización del país, que la Escuela Universitaria de Servicio Social se incorporó a la Facultad de Ciencias Sociales como "Departamento de Trabajo Social de la Facultad de Ciencias Sociales de la Universidad de la República". La consolidación de este proceso se producirá durante los gobiernos del Frente Amplio (Acosta, 2005; Ortega, 2008; Claramunt, 2018). 
Debe considerarse, sin embargo, que el cambio de mando del ejecutivo nacional en el país no se explica únicamente por articulaciones políticas. Es un proceso complejo entrelazado con la economía política, es decir, está ligado de manera decisiva a proyectos políticos que se articulan y fusionan para enfrentar tensiones estructurales afectadas por la economía dependiente, en este caso en las condiciones particulares de Uruguay. ${ }^{6} \mathrm{La}$ degradación del país, explícitamente en marcha desde 2013, se abrió de par en par en 2020, agravada por la crisis pandémica. En el ámbito de las relaciones laborales, aun considerando el carácter más intervencionista de los gobiernos frenteamplistas, se impuso una característica en el mundo y en América Latina: la relativa expansión/mantenimiento de los puestos de trabajo estuvo acompañada de su precariedad e intensificación.

Durante los 15 años del Frente Amplio se implementaron políticas focalizadas para el manejo del pauperismo extremo, generalizadas en toda América Latina, con base en los lineamientos del Banco Mundial. Se debe enfatizar, por ejemplo, la creación del "Ministerio de Desarrollo Social (MIDES)" (2005), del "Programa de Atención para la Emergencia Social (PANES)" (2005-2007 - este último responsable de la implementación de políticas focalizadas y selectivas) y el "Plan de Equidad" (2007), acciones que son ampliamente coherentes con la noción de ciudadanía neoliberal. Cabe mencionar también que, junto con importantes medidas de protección a los trabajadores (Ley de Libertad Sindical, regulación del trabajo doméstico, la jornada de 8 horas para los trabajadores rurales y la reanudación de los Consejos de Salario, - que profundizó el debate entre el movimiento sindical y el Ministerio de Trabajo -), la precariedad del trabajo se intensificó a través de la regulación de la tercerización y subcontratación de trabajadores.

6 Siguiendo la tradición latinoamericana dependiente, lo que se ha visto en Uruguay son crecimientos abruptos seguidos de caídas profundas y nuevos crecimientos cada vez más bajos y por un período corto de tiempo. Esta inestabilidad afectó igualmente al mundo del trabajo, siempre en el sentido de tensar hacia una gestión de la ley general de acumulación capitalista tendencialmente al capital constante a expensas del capital variable. Sobre esto, ver Silva (2021) y Real GDP Growth (2020). 
También se debe enfatizar la profundización de la política asistencial - en los lineamientos aquí señalados - durante el gobierno de José Mujica, con un importante papel del Consejo Nacional de Políticas Sociales (CNPS) y el compromiso de lucha por las libertades y los derechos humanos: la aprobación de la ley del aborto, la despenalización de la marihuana, el matrimonio igualitario, la innovación en la gestión de recursos humanos, la expansión y profundización de las políticas de salud sexual y reproductiva, mental y de drogas (Claramunt, 2018). También es importante destacar el énfasis que se le da a la política educativa, incluyendo la sustancial expansión, mejoramiento y descentralización de la Universidad de la República, incluyendo mejores condiciones materiales y humanas. En todo caso, las políticas impulsadas por el Frente Amplio actuaron en el sentido de articular las políticas universales tradicionales con las focalizadas, aunque estuvieran sustancialmente dedicadas a la expansión de estas últimas.

Con base en el apoyo ruralista, una amplia composición política (incluso de extrema derecha) y un discurso que reivindicaba la seguridad de las familias uruguayas (incentivando la posesión de armas, fortaleciendo las acciones policiales contra el crimen y el narcotráfico, y reformando el sistema penitenciario), el gobierno de Lacalle Pou predicaba la austeridad y la reducción del déficit fiscal, recortes del gasto público, incentivos a la iniciativa privada, optimización y modernización del estado y una contrarreforma de la seguridad social. Se reconfiguraron los Consejos de Salarios creados en 1943 por el neobatllismo, retomados en el primer gobierno de Sanguinetti y recreados por el Frente Ampla (después de un largo período, desde el gobierno de Lacalle Herrera, padre de Lacalle Pou, en el que no fueron convocados), incluyendo representantes de los trabajadores rurales, privados y funcionarios públicos, estimulando ampliamente lo que se ha caracterizado como "modernización": aumento del mercado laboral a través de la cooperación y negociación directa entre empleadores y empleados, amplio estímulo al uso de tecnologías en el entorno laboral, esencialmente flexible y emprendedora, además de priorizar el aspecto de productividad en la negociación colectiva. 
En medio del período pandémico, Lacalle Pou envió al Congreso, en abril de 2020, su proyecto de ley más importante, a través del mecanismo de "Ley de Urgente Consideración" (LUC), considerado de suma importancia para el proyecto de gobierno. Este proyecto de ley, aprobado en 2020 por el Parlamento sin cambios sustanciales, redimensionó - en la perspectiva ya descrita y a través de 476 artículos - temas importantes como educación, salud, trabajo, economía y medio ambiente, además de dar gran énfasis a el área de seguridad. Esto ha venido sucediendo no sin una fuerte resistencia sindical y de movimientos sociales progresistas, que obtuvieron las firmas necesarias para convocar un referéndum para intentar derogar 135 artículos de la ley.

\section{El Trabajo Social en Uruguay y su fracción más crítica}

Surge en este momento una pregunta: ¿cómo situar el debate crítico sobre Trabajo Social en este complejo contexto? Cabe señalar que la renovación del Trabajo Social en Uruguay en la década de 1960 y su particular inserción en el proceso de reconceptualización latinoamericano se desarrollaron en un contexto de reorganización política y económica del país desde la segunda mitad de la década de 1950 en adelante. La hegemonía tradicional colorada, sus bases históricamente centradas en acciones, propuestas y políticas marcadas por la moderación de los conflictos estructurales ingresaron en un proceso de crisis terminal. Es en este contexto que se consolidaron propuestas ampliamente liberales bajo la dirección del Partido Nacional entre 1959 y 1967, y se profundizó la polarización que culminó en un acontecimiento importante dentro de la clase obrera: la unificación sindical (1966). La polarización social se consolidó luego en dos grandes bloques en el país: por un lado, un amplio movimiento estudiantil (también unificado a través de la Federación de Estudiantes Universitarios del Uruguay), el movimiento obrero e importantes sectores de las clases medias urbanas; por otro, los sectores tradicional-conservadores liderados por el Partido Nacional 
por primera vez en la historia del país. A esto se suma un complejo escenario internacional marcado por la Guerra Fría, la intervención de Estados Unidos en la región y la Revolución Cubana y sus consecuencias en toda América Latina.

En el ámbito del Trabajo Social, se formó y gradualmente se consolidó una tradición progresista muy diversa y fuertemente militante. A partir de este complejo escenario, se articularon aquí diversos elementos que constituyeron la base que sostuvo la génesis, consolidación y desarrollo de la fracción más crítica del Servicio Social uruguayo, no sin desconocer explícitamente rasgos sociohistóricos explícitamente republicano-liberal-democráticos, seculares, con fuerte inspiración francesa-ilustrada, con la valorización de un cierto tipo de "ciudadanía hiperintegradora" y una fuerte intervención estatal. Esta amplia base social de apoyo progresista actuó decisivamente en la creación del Frente Amplio en 1971, además de enriquecerse con sectores más progresistas colorados y blancos que abandonaron su base tradicional y contribuyeron a crear una importante unidad que también se expresó en el Servicio Social Uruguayo.

La resistencia contra la dictadura militar contó con el apoyo hegemónico de los trabajadores sociales uruguayos, en su amplia diversidad. Sus distintas tendencias, aunque debatidas, convergieron en relación a la resistencia al régimen cívico-militar y a la represión del centro de formación (Acosta, 2005, p. 244-247), considerando: a) la pérdida de autonomía de la "Escuela Universitaria de Servicio Social" en 1973; b) la destitución de sus autoridades legalmente constituidas con el nombramiento de interventores que estuvieran decididos a cerrar la unidad docente; c) la exigencia de que los empleados de la escuela, a partir de 1976, firmaran la llamada "declaración de fe democrática" (!) jurando no participar en organizaciones, acciones y hechos considerados subversivos, hecho que motivó el despido de varios docentes; d) y la fuerte represión del movimiento estudiantil, que logró iniciar su reorganización en 1979, aunque de manera clandestina, a través del Centro de Estudiantes Universitarios 
de Servicio Social (más abiertamente presente a partir de 1983 con el fortalecimiento del movimiento sindical). También es importante destacar la fundación de ADASU (Asociación de Asistentes Sociales del Uruguay) en octubre de 1981 (en pleno régimen cívico-militar), producto de esta amplia reorganización y resistencia que involucró a profesionales de distintas orientaciones políticas y teóricas dentro de la profesión en ese tiempo histórico.

Otro hecho importante acentuó y marcó decisivamente la tendencia progresista del Trabajo Social en Uruguay: la Escuela Universitaria de Servicio Social fue incorporada por la Facultad de Ciencias Sociales en 1992, luego de un amplio debate sobre el sentido que debe adoptarse en la formación de área de profesionales, un debate que ya estuvo influido por los impactos de la reestructuración productiva en América Latina y Uruguay en la década de los noventa (por tanto, un momento histórico distinto a los sesenta/setenta). Cabe resaltar la importancia de insertar el Trabajo Social como disciplina con el mismo estatus atribuido a las áreas de las ciencias sociales, asociadas a ellas como área particular, destacando la relación de esta profesión con la "cuestión social", sus múltiples refracciones (en el campo de las políticas sociales), abriendo la posibilidad de formar trabajadores sociales intelectuales, es decir, productores de conocimiento como parte de un área de conocimiento.

La creación de marcos académicos favoreció la consolidación teórica, permitiendo a los docentes trabajadores sociales dedicarse plenamente a la producción de conocimiento en el campo de las Ciencias Sociales y Humanas. Este salto se consolidó con la primera maestría realizada en alianza con el Programa de Posgrado en Servicio Social de la Universidad Federal de Río de Janeiro (UFRJ) y posteriormente como maestría y doctorado autónomo con salida en Trabajo Social como parte de la Facultad de Ciencias Sociales. A esto se suma el atraso acumulado del Servicio Social en el proceso de resistencia al régimen cívico-militar y durante la reanudación de la democratización política en el país a partir de 1985. Por tanto, la disolución de la EUSS y su recreación vinculada a la 
Facultad de Ciencias Sociales de la Universidad de La República significó el fin del ciclo de los años sesenta y la reorganización de las corrientes teórico-prácticas articuladas principalmente en ese contexto, sometiendo a la crítica proyectos de inspiración desarrollista, orientación sociológico-modernizadora y educación popular (Acosta, 2005, p. 255-262).

En esta etapa se dieron años de continua expansión y consolidación de la profesión, que culminaron con los 15 años de gobierno del Frente Amplio desde 2005. Este proceso marcó un profundo cambio en el ámbito estatal, su modernización, así como la expansión asistencial, políticas con impactos en el espacio sociolaboral de esta profesión y en el ámbito de la gestión del pauperismo relativo y absoluto. También ofreció una gama de temas de estudio para los intelectuales de la zona, promoviendo producciones teóricas articuladas con las políticas sociales implementadas por el Estado uruguayo a partir de las décadas de 1990 y 2000, políticas vinculadas a estrategias de gobierno orientadas por el Banco Mundial ampliamente utilizadas en América Latina con diferentes denominaciones.

La expansión del mercado laboral —incluidos los (as) trabajadores (as) sociales - también se caracterizó por su precariedad y su brutal intensificación, que provocó - en general - la necesidad también de que estos (as) profesionales necesiten más de una fuente generadora de recursos para su sustento (Claramunt, 2018). Más que eso, hubo cambios sustanciales en la vida del pueblo uruguayo a través de propuestas que comenzaron a articular políticas y programas universales fundamentales con políticas de gestión del pauperismo extremo. Esta reingeniería también afectó a las universidades ya sea para crear y consolidar importantes funciones docentes en condiciones nunca vistas en el país, o para reestructurar estos roles dentro de la institución universitaria (Bentura; Vecinday, 2019, p. 225).

Como resultado de este amplio contexto y de las intensas luchas libradas en las condiciones particulares del país, surgió una diversa tradición teórica progresista, ubicada sobre todo en los ámbitos académicos, 
específicamente en la Universidad de la República. Sin duda, tiene un eje temático estructurante: el análisis del objeto concreto que la profesión trata cotidianamente, priorizando el estudio de las políticas sociales, siguiendo la tradición "hiperintegradora" de programas y proyectos sociales históricamente presentes en el país. Se diversifica y refracta, entonces, en diferentes temas de estudio y líneas teóricas progresistas-libertarias igualmente heterogéneas. Está políticamente articulada con la tradición político-partidaria más progresista del país, específicamente el Frente Amplio y sus diversas facciones internas.

A partir de esta base, se ha constituido una intelectualidad amplia, diversa y actualizada en la relación con las fuerzas progresistas. Ejemplos importantes y heterogéneos son: a) Laura Vecinday con estudios originales en el campo de la asistencia y protección social (Bentura; Vecinday, 2019; Venciday; Bentura, 2019); b) Alejandro Mariatti y su investigación sobre la globalización de la economía capitalista, la tecnocratización, la activación del mercado laboral y las transformaciones del trabajo en Uruguay (Bentura; Mariatti, 2018; 2016; Mariatti, 2020); c) Adela Susana Claramunt y sus importantes análisis sobre la realidad del Trabajo Social en Uruguay hoy (Claramunt, 2018); d) José Pablo Bentura y el estudio de las políticas sociales en el contexto actual de acumulación capitalista, el rol del Estado y en este el Servicio Social (Bentura; Mariatti, 2018; Bentura et al., 2016); e) Alejandro Casas y el análisis de categorías importantes extraídas de la realidad latinoamericana y uruguaya: Estado, sociedad civil, intelectuales, moralidad, orientación sociocultural estratégica, correlación de fuerzas, cultura y revolución pasiva (como el transformismo - Casas, 2018; Casas, 2019); f) Elisabeth Ortega Cerchiaro y su investigación sobre la relación entre medicina y religión, debatiendo, en este contexto, a partir del análisis genealógico de Foucault, las transformaciones del Trabajo Social en Uruguay en los veinte años anteriores a la dictadura cívico-militar del país (Ortega, 2011); g) Carolina González Laurino y Sandra Leopold Costábile, con producciones conjuntas o propias, destacan las recientes transformaciones en el modelo de protección social uruguayo, con especial énfasis en el debate sobre niñez y juventud 
(Leopold Costábile; González Laurino, 2015; González Laurino; Leopold Costábile, 2018; Leopold Costábile, 2014; González Laurino, 2021); h) Silvia Rivero con sus estudios sobre nuevas formas de gestionar las políticas sociales en asociación con las denominadas organizaciones de la sociedad civil (Rivero, 2013); i) Mónica de Martino y sus investigaciones sobre sistemas de protección social, familias, procesos de ahorro social intensificados por el discurso de vulnerabilidad social y debates de género, con énfasis en el estudio de políticas sociales dedicadas a segmentos y posibilitadas por el Estado. Su originalidad radica, sin embargo, en una postura analítica que señala lo que la autora entiende como los límites de Marx y el marxismo en el debate con Foucault y, más recientemente, con Sartre (De Martino, 2020; 2010; 2009).?

\section{Consideraciones finales}

La amplia organización de la resistencia a la dictadura cívico-militar, el proceso de redemocratización política en el país y el fin de la intervención en la Universidad fueron determinantes para que la Escuela Universitaria de Servicio Social pasara a formar parte de la Facultad de Ciencias Sociales de la UDELAR a través de un Departamento de Trabajo Social. Lo que hasta entonces fue predominantemente un proceso de movilización político-militante - profundizado por la renovación del Trabajo Social en Uruguay dentro del proceso de reconceptualización - elevó otros niveles a medida que los profesionales del área de Trabajo Social comenzaron a ocupar el espacio universitario, profundizaron su formación teórica, empezaron a realizar investigaciones y producir conocimiento. Este proceso también fue de gran valor para que los trabajadores sociales pudieran ampliar su diálogo más allá de los límites profesionales, en el área de las Ciencias Sociales, creando las condiciones objetivas para un

Carlos Montaño, Luis Acosta, Gabriela Lema y Alejandra Pastorini Corleto, aunque tengan una vida profesional en Brasil, también tienen una producción importante en el área dentro y fuera de Uruguay. 
debate más denso y maduro entre la teoría social y el legado construido objetivamente por la profesión durante años de conservadurismo, renovación, organización, resistencia, mayor proximidad a los cuerpos académicos y formativos y su consolidación como área de conocimiento.

Luego de sucesivos años de luchas y logros emprendidos por el Servicio Social Uruguayo, su explícita expansión como profesión y consolidación como área de conocimiento en el campo de las Ciencias Humanas y Sociales - en estrecha relación con los logros del país - un período de estancamiento parece imponerse, fruto de la ofensiva del capital sobre el trabajo desde las condiciones determinadas por el imperialismo y el capitalismo dependiente reorganizado en la región en el proceso de crisis estructural del capital. En esto, ciertamente hay aspectos particulares que marcan la historia reciente del país con la revitalización explícita de la derecha y extrema derecha uruguayas. Los retrocesos en el campo de las políticas sociales (no solo las de carácter más universal), las precarias condiciones laborales de los trabajadores sociales (dentro y fuera de las universidades) y las dificultades relacionadas con las condiciones necesarias para la producción de conocimiento auténtico y compromiso con la crítica radical, tienden a profundizar. Frente a esto, las facciones progresistas de Uruguay (con ella las de la profesión) tienen una tradición de la que nunca podrán abdicar: la unidad sindical, una importante tradición laico-republicana y organización partidaria y popular. Pero esto no es solitariamente: necesita expresar proyectos y alternativas de resistencia global y latinoamericana.

\section{Referencias}

ACOSTA, Luis. O processo de renovação do Serviço Social no Uruguai. 2005. Tese (Doutorado) — Universidade Federal do Rio de Janeiro, Rio de Janeiro, 2005.

BENTURA, José Pablo; MARIATTI, Alejandro. La integralidad de las funciones universitarias en el contexto de mundialización de la economía. Apuntes desde el Trabajo Social. Revista Fronteras, Montevideo: Departamento de Trabajo Social, n. 9, mar. 2016. 
BENTURA, José Pablo; MARIATTI, Alejandro. La metamorfosis del trabajo y los procesos de activación. Serviço Social \& Sociedade, São Paulo: Cortez, n. 131, p. 89-108, jan./abr. 2018.

BENTURA, José Pablo; VECINDAY, Laura. Desafios, obstáculos e tensões do Serviço Social uruguaio no início do século XXI. In: YAZBEK, Maria Carmelita; IAMAMOTO, Marilda Villela (org.). Serviço Social na história: América Latina, África e Europa. São Paulo: Cortez, 2019. p. 217-239.

BENTURA, Pablo et al. Exigência de condicionalidades: significados, alcances e controvérsias no debate. In: SILVA E SILVA, María Ozanira da (org.). O mito e a realidade no enfrentamento à pobreza na América Latina: estudo comparado de programas de transferência de renda no Brasil, Argentina e Uruguai. São Paulo: Cortez, 2016. p. 147-170.

CAETANO, Gerardo. La República Batllista. Montevideo: Ediciones de La Banda Oriental, 2015. (Colección HR).

CASAS, Alejandro. Luchas por la hegemonía y bloques históricos en el Uruguay contemporáneo y en la actual coyuntura. In: CASAS, Alejandro (org.). Sujetos colectivos populares, mundo del trabajo y territorios: estudios en el Uruguay progresista. Montevideo: Área Académica de Liberación, 2018. p. 6-40.

CASAS, Alejandro (org.). Sujetos colectivos populares, mundo del trabajo y territorios: estudios en el Uruguay progresista. Montevideo: Área Académica de Liberación, 2019.

CERCHIARO, Elizabeth Ortega. El Servicio Social y los procesos de medicalización de la sociedad uruguaya en el periodo neobatllista. Montevideo: Trilce, 2008.

CLARAMUNT, Adela S. Los trabajadores sociales en el Uruguay en la última década: sus espacios socio-ocupacionales y condiciones de trabajo. 2018. Tese (Doutorado) Universidad de la República, Montevidéu, 2018.

DE MARTINO, Mónica. Familias y protección social. Diálogos entre el campo del marxismo y Foucault. Revista de Políticas Públicas, São Luís: UFMA, v. 13, p. 43-53, 2009.

DE MARTINO, Mónica. Marxismo, género e historia social. Puntos y contrapuntos. Revista de Políticas Públicas, São Luís: UFMA, v. 14, p. 39-46, 2010.

DE MARTINO, Mónica. Sobre el método progresivo-regresivo sartreano: una perspectiva para el trabajo social. Katálysis, Florianópolis, v. 23, n. 3, p. 590-600, dez. 2020.

DE MARTINO, Monica Solange; ORTEGA, Elisabeth. Passado e presente do Serviço Social uruguaio. Sobre as teses de precarização. In: YAZBEK, Maria Carmelita; IAMAMOTO, Marilda Villela (org.). Serviço Social na história: América Latina, África e Europa. São Paulo: Cortez, 2019. p. 188-216.

FERNANDES, Florestan. Capitalismo dependente e classes sociais na América Latina. São Paulo: Global, 2009. 
GONZÁLEZ LAURINO, Carolina. Sentidos, prácticas y modificaciones en los informes técnicos del sistema penal juvenil uruguayo. Tempo Social, v. 33, n. 1, p. 203-224, 2021.

GONZÁLEZ LAURINO, Carolina; LEOPOLD COSTÁBILE, Sandra. Transformaciones en la matriz de protección uruguaya a inicios del siglo XXI. Textos \& Contextos, Porto Alegre, v. 17 , n. 1, p. 52-66, 2018.

KATZ, Claudio. A teoria da dependência: 50 anos depois. São Paulo: Expressão Popular, 2020.

LEOPOLD COSTÁBILE, Sandra. Los laberintos de la infancia: discursos, representaciones y crítica. Montevideo: Universidad de la República Uruguay; CSIC, 2014.

LEOPOLD COSTÁBILE, Sandra; GONZÁLEZ LAURINO, Carolina. Los riesgos de la individualización en el nuevo modelo de protección social uruguayo: el caso del Programa Jóvenes en Red. Sociedade em Debate, v. 21, n. 1, p. 211-254, 2015.

MARIATTI, Alejandro. El proceso de transformación laboral en Uruguay. Textos \& Contextos, Porto Alegre, v. 19, n. 1, e38359, 2020.

MARINI, Ruy M. América Latina, dependencia y globalización. Bogotá: Clacso; Siglo del Hombre Editores, 2008.

ORTEGA, Elizabeth. El Servicio Social y los procesos de medicalización de la sociedad uruguaya en el período neobatllista. Montevideo: Trilce, 2008.

ORTEGA, Elizabeth. Medicina, religión y gestión de lo social: un análisis genealógico de las transformaciones del Servicio Social en el Uruguay (1955-1973). Montevideo: Udelar, 2011.

REAL DE AZUA, Carlos. Uruguay, ¿una sociedad amortiguadora? Montevideo: Ediciones de La Banda Oriental, 1984. (Colección Estudios sobre la Sociedad Uruguaya, número 3).

REAL GDP GROWTH. International Monetary Fund. [2020]. Disponível em: https://www. imf.org/external/datamapper/NGDP_RPCH@WEO/OEMDC/ADVEC/WEOWORLD. Acesso em: 28 jul. 2020.

RIVERO, Silvia. De la acción política a la gestión: sociedad civil en movimiento. 2013. Tese (Doutorado) - Facultad de Ciencias Sociales, Universidad de la República, Montevideo, 2013.

SILVA, José Fernando S. América Latina: capital e devastação social. Katálysis, Florianópolis: UFSC, v. 24, n. 1, 2021.

VENCINDAY, Laura; BENTURA, José Pablo (org.). Entre la asistencia y la activación: intervenciones sobre la pobreza en el Uruguay progresista. Montevideo: Udelar; Grupo de Estudios sobre Sistemas de Protección Social, Prácticas Institucionales e Profesionales, 2019. 


\section{Sobre os autores}

José Fernando SiqueIRa da Silva - Livre-docente em Serviço Social pela Universidade Estadual Paulista (Unesp). Pós-doutor em Serviço Social pela Universidade Nacional de La Plata (UNLP — Argentina) e Universidade da República (Udelar - Uruguai). Professor associado. Bolsista Produtividade do CNPq desde março de 2009 (nível 2). Coordenador do Grupo de Estudos e Pesquisas Marxistas (Gepem).

E-mail: jose.siqueira-silva@unesp.br

José Pablo Bentura - Doutor em Ciências Sociais pela Faculdade de Ciências Sociais (FCS) da Universidade da República (Udelar - Uruguai). Docente e pesquisador. Pesquisador do Sistema Nacional de Investigadores da Agência Nacional de Investigação e Inovação (ANII).

E-mail: pbentura@gmail.com 